\title{
Fluoroquinolone Resistance Mechanism of Clinical Isolates and Selected Mutants of Pasteurella multocida from Bovine Respiratory Disease in China
}

\author{
Ling-Cong $\mathrm{KONG}^{1}$, Duo $\mathrm{GAO}^{1)}$, Yun-Hang $\mathrm{GAO}^{1)}$, Shu-Ming LIU ${ }^{1)}$ and Hong-Xia MA ${ }^{1) *}$ \\ ${ }^{1)}$ College of Animal Science and Technology, Jilin Agricultural University, Xincheng Street No.\#2888, Changchun 130118, P.R. China
}

(Received 9 May 2014/Accepted 14 August 2014/Published online in J-STAGE 17 September 2014)

\begin{abstract}
The minimum inhibitory concentrations (MICs), mutation prevention concentrations (MPCs) and contribution of quinolone resistance-determining region (QRDR) mutations to fluoroquinolone (ciprofloxacin, enrofloxacin and orbifloxacin) susceptibility in 23 Pasteurella multocida (Pm) isolates were investigated. Fluoroquinolone-susceptible isolates (MICs $\leq 0.25 \mu \mathrm{g} / \mathrm{ml}, 9$ isolates) had no QRDR mutations, and their respective MPCs were low. Fluoroquinolone-intermediate isolates (MICs $=0.5 \mu \mathrm{g} / \mathrm{m} l, 14$ isolates) had QRDR mutations (Asp87 to Asn or Ala84 to Pro in gyrA), and their respective MPCs were high (4-32 $\mu \mathrm{g} / \mathrm{m} l$ ). First-step mutants ( $\mathrm{n}=5$ ) and laboratory-derived highly resistant fluoroquinolone mutants $(\mathrm{n}=5)$ also had QRDR mutations. The MICs of fluoroquinolones for mutant-derived strains were decreased in the presence of efflux inhibitors. The results indicated that the fluoroquinolone resistance of $P m$ is mainly due to multiple target gene mutations in gyrA and parC and the overexpression of efflux pump genes.

KEY WORDS: efflux pump, fluoroquinolone resistance, Pasteurella multocida, plasmid, target mutation
\end{abstract}

doi: 10.1292/jvms.14-0240; J. Vet. Med. Sci. 76(12): 1655-1657, 2014

Recent studies have suggested that one of the important pathogens of bovine respiratory disease (BRD) that seriously harm cattle in China and other countries is Pasteurella multocida $(P m)$ of Serogroup A $[4,6,10]$. A Pm infection is treated mainly using antibiotics $[5,11]$. In recent years, fluoroquinolone antibiotics including ciprofloxacin (CIP) and enrofloxacin (ENR) have been increasingly used to treat $\mathrm{Pm}$ infections in China. Fluoroquinolones at subinhibitory concentrations lead to emergence of resistant bacterial strains [13]. Hence, fluoroquinolones at subinhibitory antimicrobial concentrations pose a major risk in the treatment of BRD. Fluoroquinolone resistance has been described for Mannheimia haemolytica and Mycoplasma bovis isolates from BRD [7, 12, 14]. However, fluoroquinolones resistance of $\mathrm{Pm}$ isolates has very rarely been studied. In the present study, the potential risk of fluoroquinolone resistance of $\mathrm{Pm}$ isolates was evaluated by determining the mutation prevention concentrations (MPCs) of fluoroquinolones, mutationmediated alterations in quinolone resistance-determining regions (QRDRs), fluoroquinolone resistance plasmid and efflux-mediated mutational changes in $\mathrm{Pm}$ isolates obtained from various cattle farms in China.

Twenty-three field isolates of Pm (designated Pm1 to Pm 23) were obtained from cattle lungs from 23 farms located in different provinces of China during the period of 2011 to 2013. All isolates and capsule serotypes were identified as

*Correspondence to: Ma, H.-X., College of Animal Science and Technology, Jilin Agricultural University, Xincheng Street No.\#2888, Changchun 130118, P.R. China.

e-mail: hongxia0731001@163.com

(c)2014 The Japanese Society of Veterinary Science

This is an open-access article distributed under the terms of the Creative Commons Attribution Non-Commercial No Derivatives (by-nc-nd) License $<$ http://creativecommons.org/licenses/by-nc-nd/3.0/>. described previously [17]. Antimicrobial susceptibility testing with ENR, CIP and orbifloxacin (ORB) was performed using the broth microdilution method, as recommended by the Clinical and Laboratory Standards Institute guidelines in VET01-A4 [2]. The reference strain Escherichia coli ATCC 25922 served as an internal control. The method for measuring MPC values has been previously described [3]; the lowest drug concentration that prevented the emergence of mutants after a 5-day incubation period was recorded as the MPC, and the values for mutant selection windows (MSWs) were calculated. Each experiment was repeated two times. A mutant of each original strain (Pm1-Pm23) was randomly selected from plates with a concentration of ENR, CIP and ORB that was one dilution (i.e., twofold) lower than the MPC (sub-MPC). Each mutant was cultured on antimicrobial-free agar plates for 3 serial passages, and then, colonies were tested for fluoroquinolone susceptibility. The minimum inhibitory concentrations (MICs) of mutants were measured using the agar dilution method [1], and MICs of mutants higher than those of the parent isolates confirmed the existence of mutants. In vitro-derived highly resistant fluoroquinolone mutants of $P m$ were obtained from wildtype (Pm-8, Pm-9, Pm-16 and Pm-20) and Type II (Pm-3) isolates of parent strains through serial inoculations in brain heart infusion (BHI, Oxoid Ltd., Cambridge, U.K.) agar plates containing ENR at a subinhibitory concentration. The suspension of bacterial inoculums in BHI agar was adjusted to $0.5 \mathrm{McFarland}$ standards. Then, $200 \mu \mathrm{l}$ of this suspension was added into a tube containing BHI medium $(1,800 \mu \mathrm{l} /$ tube) with twofold serial dilutions of ENR (final concentration ranging from 0.06 to $128 \mu \mathrm{g} / \mathrm{m} l$ ). The tube was visually examined for bacterial growth, and the respective MICs were noted. The bacteria with the highest MIC were harvested and dispersed $(100 \mu l /$ tube $)$ in a new tube containing BHI medium $(1,900 \mu \mathrm{l} /$ tube $)$ with twofold serial 
Table 1. QRDR mutation genotypes and fluoroquinolone MICs and MPCs for Pm field strains

\begin{tabular}{|c|c|c|c|c|c|c|c|c|c|}
\hline \multirow{2}{*}{$\begin{array}{c}\text { Mutation } \\
\text { type }\end{array}$} & \multirow{2}{*}{$\begin{array}{l}\text { Number of } \\
\text { strains }\end{array}$} & \multicolumn{3}{|c|}{ MIC range $(\mu \mathrm{g} / \mathrm{m} l)$} & \multicolumn{2}{|c|}{ Substitution in } & \multicolumn{3}{|c|}{ MPC range $\left.^{\text {a) }}(\mu \mathrm{g} / \mathrm{m} l) / \mathrm{MSW}^{\mathrm{b}}\right)$} \\
\hline & & CIP & ENR & ORB & gyr A & parC & CIP & ENR & ORB \\
\hline Wild & 9 & $0.03-0.25$ & $0.03-0.25$ & $0.03-0.06$ & None & None & $\begin{array}{c}0.125-0.5 / \\
0.06-0.25\end{array}$ & $\begin{array}{c}0.125-0.5 / \\
0.06-0.25\end{array}$ & $\begin{array}{c}0.125-0.5 / \\
0.06-1.9\end{array}$ \\
\hline I & 13 & 0.5 & 0.5 & $0.25-0.5$ & Asp87 to Asn & None & $\begin{array}{c}4-16 / \\
3.5-15.5\end{array}$ & $\begin{array}{c}8-32 / \\
3.5-31.5\end{array}$ & $\begin{array}{c}4-32 / \\
3.5-31.5\end{array}$ \\
\hline II & 1 & 0.5 & 0.5 & 0.5 & Ala84 to Pro & None & $\begin{array}{l}4 / \\
3.5\end{array}$ & $\begin{array}{c}16 / \\
15.5\end{array}$ & $\begin{array}{c}32 / \\
31.5\end{array}$ \\
\hline
\end{tabular}

a) MPCs, mutant prevention concentrations were determined on Mueller-Hinton plates for ciprofloxacin (CIP), enrofloxacin (ENR) and orbifloxacin (ORB). b) MSW, mutant selection window (antibiotic concentration found between the minimum inhibitory concentration and MPC).

Table 2. QRDR mutation genotypes and fluoroquinolone MICs in Pm-derived strains

\begin{tabular}{|c|c|c|c|c|c|c|c|c|c|}
\hline \multirow{2}{*}{$\begin{array}{l}\text { Mutation } \\
\text { type }\end{array}$} & \multirow{2}{*}{$\begin{array}{c}\text { Number of } \\
\text { strains }\end{array}$} & \multicolumn{4}{|c|}{ MIC range $(\mu \mathrm{g} / \mathrm{m} l)$} & \multicolumn{4}{|c|}{ Substitution (s) in } \\
\hline & & CIP & ENR & ORB & $\mathrm{CIP}+\mathrm{CCCP}$ & gyrA & gyr B & $\operatorname{par} \mathrm{C}$ & parE \\
\hline $\mathrm{III}^{\mathrm{a})}$ & 1 & 4 & 2 & 1 & 2 & Asp87 to Asn & Pro415 to $\mathrm{Thr}$ & Glu84 to Lys & None \\
\hline$I^{\mathrm{a})}$ & 4 & $8-16$ & $8-16$ & $4-8$ & $2-4$ & Ser83 to Ile Asp87 to Asn & None & Ser80 to Leu & None \\
\hline$V^{b)}$ & 2 & 128 & 256 & 128 & 64 & Ser83 to Ile Asp87 to Asn & Asp426 to Asn & Glu84 to Lys & None \\
\hline $\mathrm{VI}^{\mathrm{b})}$ & 3 & 128 & $64-256$ & $64-128$ & $16-64$ & Ser83 to Ile Asp87 to Asn & None & Ser80 to Leu Glu84 to Lys & None \\
\hline
\end{tabular}

a) First-step mutant strains. b) Highly-resistant mutant strains selected using ENR.

dilutions of ENR as described above (final concentration was $128 \mu \mathrm{g} / \mathrm{ml}$ ). Then, the mutant's colonies were randomly picked up and subjected to MIC determination. The mutant colonies were then subcultured (seven subcultures) in antibiotic-free BHI medium to assess the stability of the mutant strains. The primers of QRDRs of gyrA (F, 5'-CCTTATCGTAAATCCGCTCGTA-3'; R, 5'-CGCAGGGACTTTGGTTGGGAG-3'), gyrB (F, 5'-GAAATGACCCGCCGTAA-3'; R, 5'-CTTGCCTTTCTTCACTTTGTA-3'), $\operatorname{parC}$ (F, 5'-TACGAAGGCATTGAACAAACCC-3'; R, 5'-CACTGTCCCTTGCCCTAACTC-3') and parE (F, 5'-CTAAAAGATTTGGAGCCAGTG-3'; R, 5'-TTATACGGCTAAATCCACCTGT-3') were designed according to the published GenBank deoxyribonucleic acid sequences (accession No. CP003022.1). Protocols for amplification of $q n r \mathrm{~A}, q n r \mathrm{~B}, q n r \mathrm{C}, q n r \mathrm{D}, q e p \mathrm{~A}, o q x \mathrm{~A}, o q x \mathrm{~B}$ and $a a c\left(6^{\prime}\right)-\mathrm{Ib}$ genes have been previously described [16]. The MIC of CIP was determined in the presence of potent efflux inhibitors, carbonyl cyanide m-chlorophenyl hydrazones (CCCPs) at appropriate concentrations (MIC/2) using the broth microdilution method. The $P m$ strains with a fourfold or greater reduction in their MICs in the presence of inhibitors were considered positive for CCCP efflux [15]. Each experiment was repeated three times.

The QRDR mutation types and fluoroquinolone MICs and MPCs for Pm isolates are shown in Table 1. Nine (39.1\%) isolates (wild type) were susceptible, and the remaining 14 (60.9\%) isolates (Type I, Asp87 to Asn in gyrA; Type II, Ala84 to Pro in gyrA) were intermediate to fluoroquinolones. These may be the first-step mutations in the $P m$ isolates resistant to fluoroquinolones. The fluoroquinolone MICs for Type I and II isolates were 8- to 16-fold higher than those of the wild type, and fluoroquinolone MPCs for Type I and II isolates were 32- to 256-fold higher than those of the wild type. A previous study suggested that wild-type strains had lower mutation frequencies compared with single-mutation strains [8].

The first-step mutant colonies were randomly picked up after exposure to sub-MPC concentrations; however, only five stable mutant strains were obtained from parent strains of Type I. It was easier to get first-step mutants compared with isolates with no mutations. The results are shown in Table 2. These first-step mutants had two QRDR mutation types (Type III, Asp87 to Asn in gyrA, Pro415 to Thr in gyr B and Glu84 to Lys in parC; Type IV, Ser83 to Ile and Asp87 to Asn in gyrA and Ser80 to Leu in parC). Five highly fluoroquinolone-resistant mutants derived from wild-type and Type II isolates had two QRDR mutation types (Type V, Ser83 to Ile and Asp87 to Asn in gyrA and Glu84 to Lys in parC; Type VI, Ser83 to Ile and Asp87 to Asn in gyrA and Ser80 to Leu and Glu84 to Lys in parC). In short, amino acid substitutions at codon 83 or codon 87 in gyrA and codon 80 or codon 84 in $\operatorname{parC}$ play an important role in first-step mutant strains. Similarly, the present study results showed that the highly resistant $P m$ mutants in vitro had double mutations in gyrA (codon 83 and 87) and single mutations in $\operatorname{parC}$ (codon 84), which played a role in conferring a higher level of resistance to fluoroquinolones. These amino acid substitutions have been described already in previous reports, which showed amino acid substitutions in gyrA (codon 83, codon 84 and codon 87) and $\operatorname{parC}$ (codon 80 and codon 84$)[15,18]$. In addition, although the mutants were obtained from parent strains of Type II (Ala84 to Pro), the mutants had a substitution of Asp87 to Asn in gyrA (Types III to VI). This amino acid substitution may be reversible; however, the molecular basis of reversible actions is poorly understood.

Although plasmid-mediated quinolone resistance genes 
were not detected in any isolate, the $\operatorname{aac}\left(6^{\prime}\right)$-Ib gene was detected. The results of a previous study suggested that strains harboring a $a a c\left(6^{\prime}\right)-I b$ gene variant (aac6'-Ib-cr) showed higher quinolone resistance [9].

The mutant-derived strains were tested for CCCP-sensitive efflux. The MICs of CIP were determined using agar dilution in the presence and absence of CCCP $(4 \mu \mathrm{g} / \mathrm{ml}, \mathrm{MIC} / 2)$. The results indicated that the MIC of CIP was decreased in the presence of CCCP in mutant-derived strains. These results indicated that efflux pump genes might have played a role in the quinolone resistance of $P m$ mutants selected in vitro. The results are shown in Table 2. Sequence analysis of genome sequences of Pm(accession No. CP003022.1) showed that some genes coded for an efflux protein. However, the role of these proteins is uncertain, and this should be further investigated in future studies.

In conclusion, there are risks associated with the use of fluoroquinolone for $\mathrm{Pm}$ infections in cattle in China. The present study results suggested that for infections involving $P m$ with high MPCs, especially those containing mutations in $g y r \mathrm{~A}$ and parC genes, treatment with a combination of antimicrobials should be adopted.

Nucleotide sequence accession numbers: The nucleotide sequence data reported in this paper will appear in the GenBank nucleotide sequence databases with the following accession numbers:\#KM111304-KM111336 (gyrA), \#KM111337-KM111369 (gyrB), \#KM111370-KM111402 (parC), \#KM111403-KM111435 (parE) and \#KM111436KM111468 (aac6'-Ib).

ACKNOWLEDGMENTS. This research was funded by Important National Science Foundation (Grant No. 31272611) and the New Century Talents Project of China's Education Ministry (Grant No. NCET-10-0174).

\section{REFERENCES}

1. Chen, Y. P., Tsao, M. Y., Lee, S. H., Chou, C. H. and Tsai, H. J. 2010. Prevalence and molecular characterization of chloramphenicol resistance in Riemerella anatipestifer isolated from ducks and geese in Taiwan. Avian Pathol. 39: 333-338. [Medline] [CrossRef]

2. Clinical and Laboratory Standards Institute. 2013. Performance Standards for Antimicrobial Disk and Dilution Susceptibility Tests for Bacteria Isolated From Animals. Approved StandardFourth Edition. In: CISI document VET01-A4, CLSI, Wayne.

3. Credito, K., Kosowska-Shick, K. and Appelbaum, P. C. 2010. Mutant prevention concentrations of four carbapenems against gram-negative rods. Antimicrob. Agents Chemother. 54: 26922695. [Medline] [CrossRef]

4. Ewers, C., Lübke-Becker, A., Bethe, A., Kiebling, S., Filter, M. and Wieler, L. H. 2006. Virulence genotype of Pasteurella multocida strains isolated from different hosts with various disease status. Vet. Microbiol. 114: 304-317. [Medline] [CrossRef]

5. Kadlec, K., Brenner Michael, G., Sweeney, M. T., Brzuszkiewicz, E., Liesegang, H., Daniel, R., Watts, J. L. and Schwarz, S. 2011. Molecular basis of macrolide, triamilide, and lincosamide resistance in Pasteurella multocida from bovine respiratory disease. Antimicrob. Agents Chemother. 55: 2475-2477. [Medline] [CrossRef]
6. Katsuda, K., Hoshinoo, K., Ueno, Y., Kohmoto, M. and Mikami, O. 2013. Virulence genes and antimicrobial susceptibility in Pasteurella multocida isolates from calves. Vet. Microbiol. 167: 737-741. [Medline] [CrossRef]

7. Katsuda, K., Kohmoto, M., Mikami, O. and Uchida, I. 2009. Antimicrobial resistance and genetic characterization of fluoroquinolone-resistant Mannheimia haemolytica isolates from cattle with bovine pneumonia. Vet. Microbiol. 139: 74-79. [Medline] [CrossRef]

8. Li, Q., Bi, X., Diao, Y. and Deng, X. 2007. Mutant-prevention concentrations of enrofloxacin for Escherichia coli isolates from chickens. Am. J. Vet. Res. 68: 812-815. [Medline] [CrossRef]

9. Ma, J., Zeng, Z., Chen, Z., Xu, X., Wang, X., Deng, Y., Lü, D., Huang, L., Zhang, Y., Liu, J. and Wang, M. 2009. High prevalence of plasmid-mediated quinolone resistance determinants qnr, aac $\left(6^{\prime}\right)$-Ib-cr, and qepA among ceftiofur-resistant Enterobacteriaceae isolates from companion and food-producing animals. Antimicrob. Agents Chemother. 53: 519-524. [Medline] [CrossRef]

10. Michael, G. B., Eidam, C., Kadlec, K., Meyer, K., Sweeney, M. T., Murray, R. W., Watts, J. L. and Schwarz, S. 2012. Increased MICs of gamithromycin and tildipirosin in the presence of the genes erm(42) and msr(E)-mph(E) for bovine Pasteurella multocida and Mannheimia haemolytica. J. Antimicrob. Chemother. 67: 1555-1557. [Medline] [CrossRef]

11. Michael, G. B., Kadlec, K., Sweeney, M. T., Brzuszkiewicz, E., Liesegang, H., Daniel, R., Murray, R. W., Watts, J. L. and Schwarz, S. 2012. ICEPmu1, an integrative conjugative element (ICE) of Pasteurella multocida: analysis of the regions that comprise 12 antimicrobial resistance genes. J. Antimicrob. Chemother. 67: 84-90. [Medline] [CrossRef]

12. Ozawa, M., Asai, T. and Sameshima, T. 2009. Mutations in GyrA and ParC in fluoroquinolone-resistant Mannheimia haemolytica isolates from cattle in Japan. J. Vet. Med. Sci. 71: 493-494. [Medline] [CrossRef]

13. Ribera, A., Jurado, A., Ruiz, J., Marco, F., Del Valle, O., Mensa, J., Chaves, J., Hernández, G., Jiménez de Anta, M. T. and Vila, J. 2002. In vitro activity of clinafloxacin in comparison with other quinolones against Stenotrophomonas maltophilia clinical isolates in the presence and absence of reserpine. Diagn. Microbiol. Infect. Dis. 42: 123-128. [Medline] [CrossRef]

14. Sato, T., Okubo, T., Usui, M., Higuchi, H. and Tamura, Y. 2013. Amino acid substitutions in GyrA and ParC are associated with fluoroquinolone resistance in Mycoplasma bovis isolates from Japanese dairy calves. J. Vet. Med. Sci. 75: 1063-1065. [Medline] [CrossRef]

15. Shin, S. B., Yoo, M. H., Jeong, J. B., Kim, Y. M., Chung, J. K., Huh, M. D., Komisar, J. L. and Jeong, H. D. 2005. Molecular cloning of the $g y r \mathrm{~A}$ gene and characterization of its mutation in clinical isolates of quinolone-resistant Edwardsiella tarda. Dis. Aquat. Organ. 67: 259-266. [Medline] [CrossRef]

16. Sun, N., Liu, J. H., Yang, F., Lin, D. C., Li, G. H., Chen, Z. L. and Zeng, Z. L. 2012. Molecular characterization of the antimicrobial resistance of Riemerella anatipestifer isolated from ducks. Vet. Microbiol. 158: 376-383. [Medline] [CrossRef]

17. Townsend, K. M., Frost, A. J., Lee, C. W., Papadimitriou, J. M. and Dawkins, H. J. 1998. Development of PCR assays for species- and type-specific identification of Pasteurella multocida isolates. J. Clin. Microbiol. 36: 1096-1100. [Medline]

18. Vila, J., Ruiz, J., Goñi, P., Marcos, A. and Jimenez de Anta, T. 1995. Mutation in the gyrA gene of quinolone-resistant clinical isolates of Acinetobacter baumannii. Antimicrob. Agents Chemother. 39: 1201-1203. [Medline] [CrossRef] 